\title{
Meta-analysis of the prognostic value of C-C chemokine receptor type 7 in patients with solid tumors
}

\author{
Guangchen $\mathrm{Zu}^{1, *}$ \\ Baoyang Luo ${ }^{2, *}$ \\ Yong Yang' \\ Yuwei Tan' \\ Tianyu Tang' \\ Yue Zhang' \\ Xuemin Chen' \\ Donglin Sun'
}

'Department of Hepatobiliary and Pancreatic Surgery, The Third

Affiliated Hospital of Soochow

University, Changzhou 213003,

People's Republic of China;

${ }^{2}$ Department of Hepatobiliary Surgery,

Taizhou People's Hospital, Taizhou

225300, People's Republic of China

*These authors contributed equally to this work
This article was published in the following Dove Medical Press journal: Cancer Management and Research

Background: Expression of C-C chemokine receptor type 7 (CCR7) is associated with the prognosis of several cancers. The aim of this study was to conduct the meta-analysis to determine the prognostic value of CCR7 expression in solid tumors.

Materials and methods: We searched for relevant literature in the PubMed, Embase, and Cochrane Library databases (last updated on January 15, 2018). The associations of CCR7 expression with overall survival (OS), disease-free survival (DFS), recurrence-free survival (RFS), progress-free survival (PFS), and disease-specific survival (DSS) were estimated.

Results: In total, 30 qualified studies including 3,413 patients were enrolled. The results revealed that higher expression of CCR7 predicted poorer OS (pooled $\mathrm{HR}=1.79 ; 95 \% \mathrm{CI}=1.49-2.16$; $P<0.001$ ) and PFS (pooled HR $=2.18 ; 95 \% \mathrm{CI}=1.49-3.18 ; P<0.001$ ), but was not associated with DFS (pooled HR $=1.69 ; 95 \% \mathrm{CI}=0.79-3.61 ; P=0.175$ ), RFS (pooled HR $=1.29 ; 95 \% \mathrm{CI}$ $=0.48-3.44 ; P=0.618$ ), or DSS (pooled HR $=3.06 ; 95 \% \mathrm{CI}=0.38-24.83 ; P<0.294$ ).

Conclusion: From this meta-analysis, we concluded that high expression of CCR7 in tumor tissue is associated with poor survival in patients with solid tumors, and may be a prognostic biomarker for tumor progression.

Keywords: CCR7, solid tumors, prognosis, systematic review, meta-analysis

\section{Introduction}

Cancer remains a worldwide medical problem, and has seriously threatened human health. In 2016, there were $\sim 1.7$ million new cancer cases in the USA, and about 0.6 million cancer-related deaths. ${ }^{1}$ Although the number of cancer survivors are increasing because of early diagnosis and treatment, traditional diagnostic and therapeutic approaches still cannot control cancer progression and development. ${ }^{2}$ Thus, further evidence-based resources for cancer diagnosis are needed so that cancer patients' prognoses can be determined early and precisely.

Chemokines are a class of small molecule proteins that bind to the corresponding cell surface receptors and participate in a variety of physiological, pathological, and immunological processes. Nearly 50 genes in humans encode chemokine ligands and $>20$ corresponding chemokine receptors. Chemokines can be divided into four categories based on structural differences: CXC, CC, CX3C, and C. ${ }^{3}$ Recently, CXC chemokines and their receptors have aroused widespread attention in the field of tumor biology. Müller et al first reported the abnormal expression of $\mathrm{C}-\mathrm{C}$ chemokine receptor type 7 (CCR7, a member of the C-C chemokine receptor family) in breast cancer. ${ }^{4}$ CCR7 is the major receptor for chemokine C ligand 19 (CCL19) and chemokine C
Correspondence: Xuemin Chen; Donglin

Sun

Pancreatic Surgery, The Third Affiliated

Hospital of Soochow University, No. 185,

Juqian Street, Changzhou 21300 , People's

Republic of China

Tel +86 I38 I358 7333; +86 I35 06I I

9453

Email tomuer@I26.com; czsdI@sina.com 
ligand 21 (CCL21). ${ }^{5,6}$ It is mainly expressed on B cells, naive $\mathrm{T}$ cells, memory $\mathrm{T}$ cells, and mature dendritic cells. The binding between CCR7 and CCL19/CCL21 is vital for lymphocyte cell trafficking and homing to lymph nodes. ${ }^{7,8}$ CCR7 is also highly expressed by various malignant tumors, and contributes to biological processes such as tumor cell proliferation, ${ }^{9}$ invasion, metastasis, ${ }^{10}$ and angiogenesis. ${ }^{11}$ Thus, it may promote tumor progression and worsen patients' prognoses.

Elevated CCR7 expression has been associated with poor prognosis in patients with gastric cancer, ${ }^{12}$ esophageal squamous cell carcinoma (ESC), ${ }^{13}$ pancreatic cancer, ${ }^{14}$ and renal cell carcinoma. ${ }^{15}$ However, some studies have revealed no significant association between CCR7 expression and the outcomes of cancers like breast cancer ${ }^{16}$ and squamous cell carcinoma of the head and neck (SCCHN). ${ }^{17}$ In fact, in colorectal cancer ${ }^{18}$ and lung cancer, ${ }^{19}$ higher CCR7 expression has been found to predict better prognosis. Therefore, the prognostic value of CCR7 in cancer patients remains unclear. We conducted this systematic review and meta-analysis to further define the relationship between the expression of CCR7 and the survival outcomes of cancer patients.

\section{Materials and methods}

The guidelines of the PRISMA ${ }^{20}$ were followed in this meta-analysis.

\section{Search strategy}

We carefully search for literature in PubMed, Embase, and the Cochrane Library (last updated on January 15, 2018). Key terms used in the search were "CCR7", "C-C chemokine receptor type 7”, "receptors", “cancer”, “tumor”, “neoplasm”, "carcinoma”, "survival”, "outcome”, "prognosis", and "prognostic". These key terms were combined by the Boolean operators "OR" and "AND". Advanced limitations were not imposed during the database search. We also manually screened the reference lists of the full-text studies to avoid the possible omission of applicable studies. Two authors ( $\mathrm{Zu} \mathrm{G}$. and Luo B.) independently conducted the exhaustive research.

\section{Inclusion and exclusion criteria}

Manuscripts were considered to be eligible for inclusion in this meta-analysis if they met the following criteria: 1) solid tumors were evaluated; 2) the expression of CCR7 was measured in tumor tissue; 3 ) the relationship between CCR7 levels and patients' survival outcomes was investigated; and 4) sufficient data were provided to determine the HR and 95\% CI. Articles were excluded according to the following criteria: 1) case reports, reviews, letters, comments, conference abstracts, and experimental studies; 2) investigation of multiple factors rather than CCR7 alone; and 3) lack of key information on patients' survival outcomes, or lack of valid/ sufficient data with which to estimate the HR and 95\% CI. Two reviewers independently carried out the enrollment and exclusion based on the above criteria, and any disagreement was resolved by discussion.

\section{Quality assessment}

We evaluated the quality of each study according to the critical review checklist of the Dutch Cochrane Center proposed by the Meta-analysis of Observational Studies in Epidemiology group. ${ }^{21}$ The main standards were: 1) sufficient report on the study population and the cancer; 2) clear methods of study design; 3) explicit definition of outcome assessment; 4) detailed description of CCR7 measurement; and 5) sufficient period of follow-up. Studies that failed to meet these five standards were excluded.

\section{Data extraction}

Two researchers independently extracted valid data and related information from the eligible studies. Relevant parameters included the first author's surname, publication year, population origin, tumor type, tumor stage, sample number, detection method, follow-up period, sample collection time, cutoff values, and HRs of CCR7 expression for overall survival (OS), disease-free survival (DFS), progress-free survival (PFS), recurrence-free survival (RFS), and diseasespecific survival (DSS), and the corresponding 95\% CI and $P$-value. If a study reported the results of both univariate and multivariate analyses, the latter was selected because it accounts for confounding factors.

\section{Statistical analysis}

CCR7 levels were defined based on the cutoff value provided in each article. We used the HRs and 95\% CIs to calculate the pooled data, and thus evaluated the influence of elevated CCR7 levels on patients' prognoses. Statistical significance was defined as $P<0.05$. The $Q$ test and $I^{2}$ statistic were used to evaluate heterogeneity. If $P<0.05$ or $I^{2}>50 \%$, indicating the existence of heterogeneity, a random-effects model was used, while a fixed-effects model was used when $P \geq 0.05$ or $I^{2} \leq 50 \%$. Sensitivity, subgroup, and meta-regression analyses were used to explore the potential sources of heterogeneity. Publication bias was analyzed with a funnel plot and Begg's and Egger's tests. We used Stata version 12.0 (StataCorp LP, College Station, TX, USA) to carry out all these analyses. 


\section{Results}

\section{Study characteristics}

Using the search strategy described in the section "Materials and Methods", we initially collected 1,482 articles. After screening the titles, abstracts, and full texts of all the publications, we deemed 109 records eligible for full-text review. Of these, 79 full-text articles were excluded for having insufficient data. Thus, 30 qualified articles were enrolled in this meta-analysis ${ }^{12-19,22-43}$ (Figure 1).

The main characteristics of the included studies are summarized in Table 1. The 3,413 patients included in these studies had been diagnosed with ESC, gastric cancer, breast cancer, non-small-cell lung cancer (NSCLC), colorectal cancer, pancreatic cancer, epithelial ovarian carcinoma, cervical cancer, etc. The studies included 3,237 patients with OS data, 406 patients with DFS data, 261 patients with RFS data, 192 patients with PFS data, and 203 patients with DSS data, who were from People's Republic of China, Japan, Germany, France, USA, UK, Austria, and Korea. Twenty-two studies (73\%) reported on Asians, and eight studies (27\%) reported on Caucasians. HRs and 95\% CIs were reported directly in ten studies. CCR 7 expression in tumor tissues was measured by immunohistochemistry in all 30 studies, although the cutoff values differed among these studies.

\section{CCR7 and OS}

OS data were reported in 28 of the studies. ${ }^{12-19,22-41}$ The detailed results of the meta-analysis are listed in Table 2. As the meta-analysis results exhibited obvious heterogeneity
( $I^{2}=67.8 \% ; P=0.000$ ), we used a random-effects model to calculate the pooled HR. Higher expression of CCR7 was associated with significantly poorer OS (pooled $\mathrm{HR}=1.79$; 95\% CI $=1.49-2.16 ; P<0.001$ ) (Figure 2).

We then carried out subgroup analyses according to tumor type, tumor source, patient ethnicity, analysis type, and HR acquisition method; the detailed data are listed in Table 2. Due to the obvious heterogeneity in each group, we used random-effects models to calculate the pooled HRs and 95\% CIs. The forest plot of the study-specific pooled HRs and $95 \%$ CIs for OS according to tumor type is shown in Figure 3. Higher expression of CCR7 predicted poorer OS in patients with $\mathrm{ESC}$ (pooled $\mathrm{HR}=2.21 ; 95 \% \mathrm{CI}=1.74-2.82$; $P<0.001$ ) and gastric cancer (pooled HR $=1.99 ; 95 \% \mathrm{CI}$ $=1.58-2.51 ; P<0.001)$, but was not significantly associated with $\mathrm{OS}$ in patients with breast cancer (pooled $\mathrm{HR}=1.24$; 95\% CI $=0.94-1.62 ; P=0.123$ ), lung cancer (pooled HR $=0.89 ; 95 \% \mathrm{CI}=0.29-2.71 ; P=0.841)$, or colorectal cancer (pooled HR $=0.92 ; 95 \% \mathrm{CI}=0.20-4.30 ; P=0.919$ ). We also performed a subgroup analysis to evaluate the influence of the tumor source on OS. In patients with tumors of the digestive system and urogenital system, higher CCR7 levels were associated with poorer OS, with pooled HRs of $1.89(95 \%$ $\mathrm{CI}=1.50-2.38 ; P<0.001)$ and $2.42(95 \% \mathrm{CI}=1.87-3.13$; $P<0.001$ ), respectively (Table 2). Remarkably, in the subgroup analysis according to ethnicity, higher CCR7 levels were associated with significantly poorer OS in Asian patients (pooled HR $=1.89 ; 95 \% \mathrm{CI}=1.56-2.29 ; P<0.001$ ), but not in Caucasian patients (pooled HR $=1.53 ; 95 \% \mathrm{CI}=0.89-2.62$; $P=0.121$ ) (Table 2).

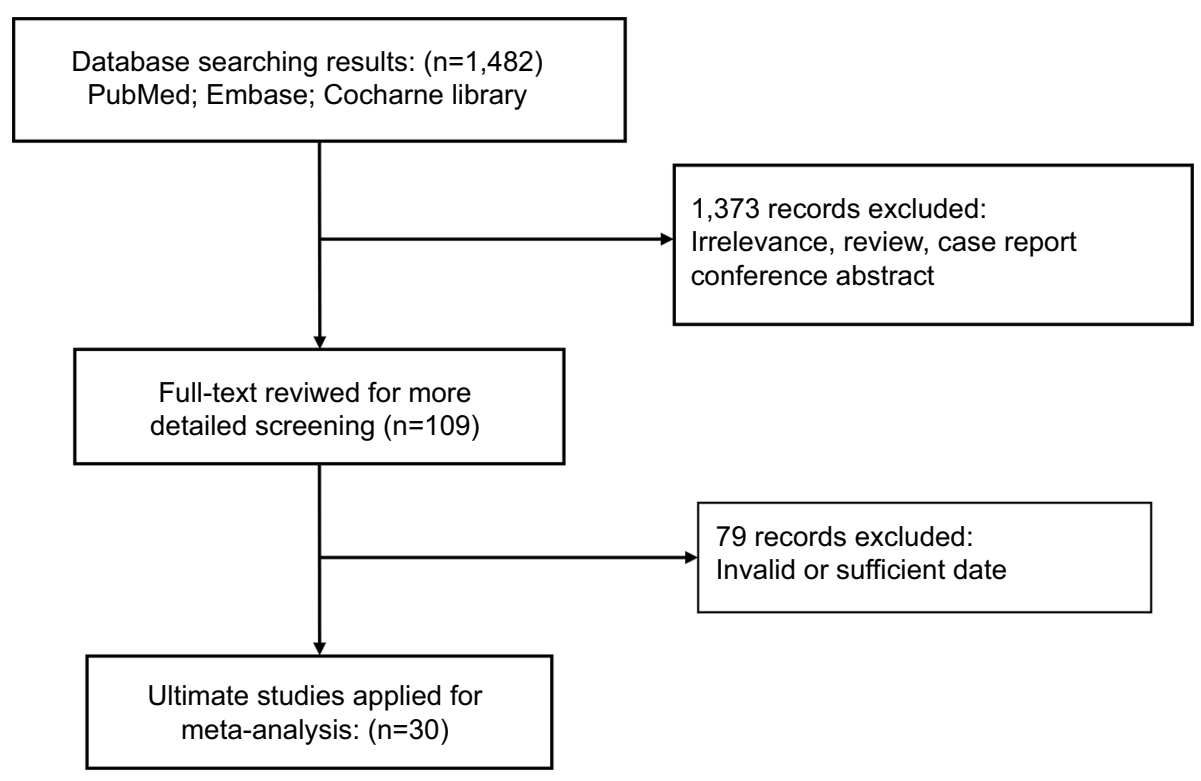

Figure I Flowchart of the study selection process. 


\begin{tabular}{|c|c|c|c|c|c|c|c|}
\hline 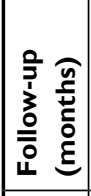 & 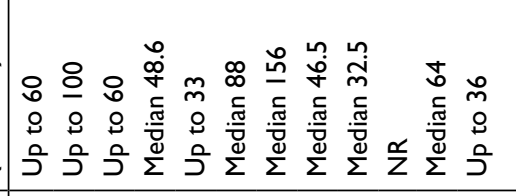 & 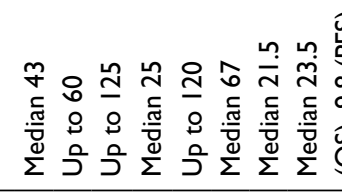 & 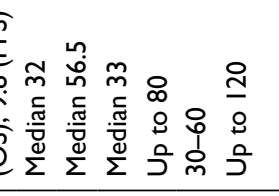 & & & 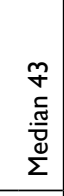 & \\
\hline$\frac{\mathscr{q}}{I}$ & 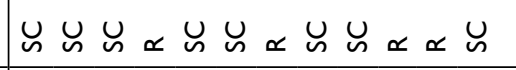 & $\propto u \cup u$ & 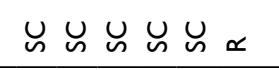 & u & $\propto \propto$ & u & \\
\hline 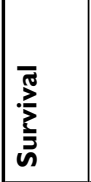 & 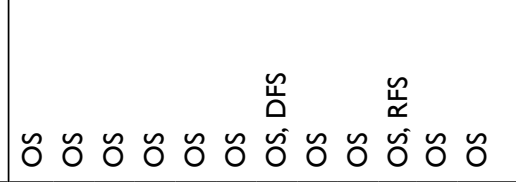 & 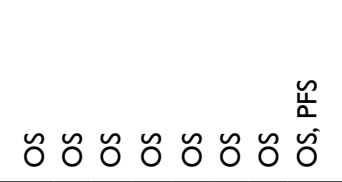 & 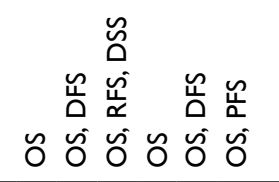 & 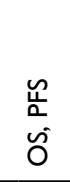 & 乞ั ڤ̆ & $\underset{\stackrel{u}{x}}{\breve{c}}$ & \\
\hline 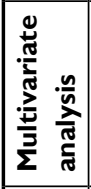 & 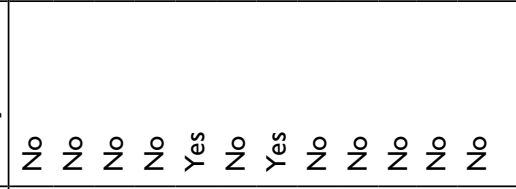 & 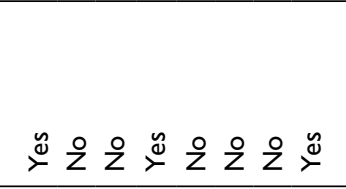 & 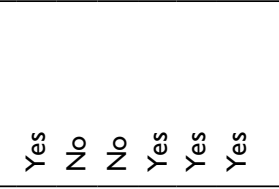 & 운 & $\stackrel{y}{=} \stackrel{0}{z}$ & $\stackrel{\circ}{z}$ & \\
\hline 惫 & 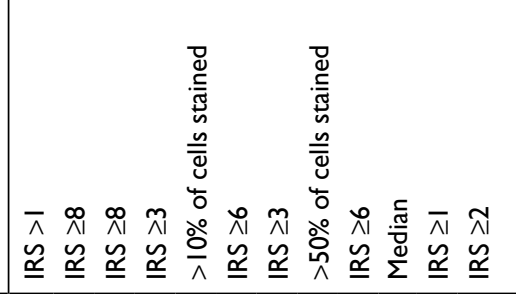 & 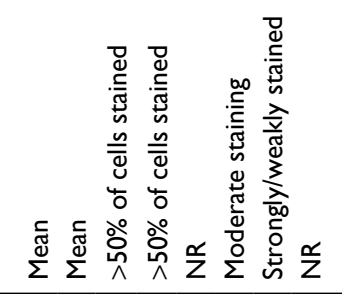 & 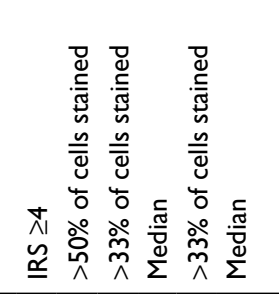 & $\stackrel{\alpha}{z}$ & 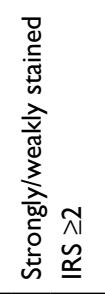 & $\begin{array}{l}\stackrel{\Delta}{\hat{N}} \\
\underline{\underline{\underline{N}}}\end{array}$ & \\
\hline 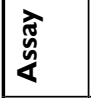 & 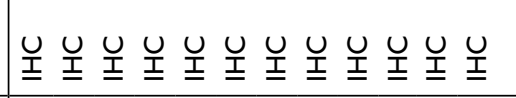 & 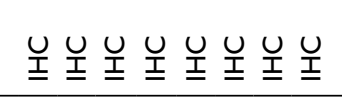 & 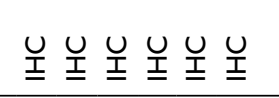 & 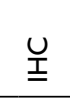 & $\stackrel{\underline{\underline{I}}}{\underline{\underline{U}}} \underline{\underline{\underline{I}}}$ & $\underline{\underline{\underline{U}}}$ & \\
\hline हूँ & 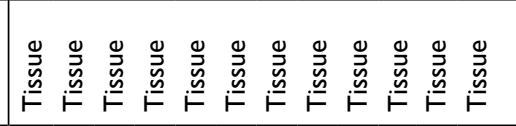 & 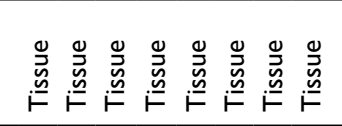 & 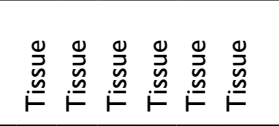 & $\stackrel{0}{\overrightarrow{\underline{\underline{w}}}}$ & 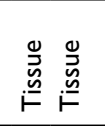 & 离 & \\
\hline 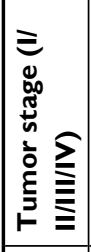 & 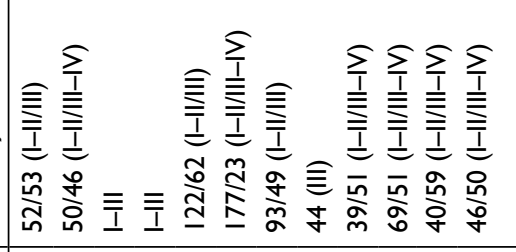 & 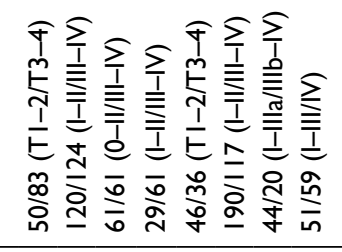 & 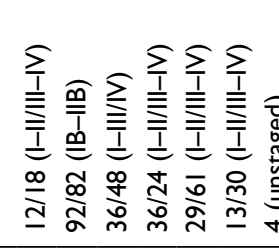 & & 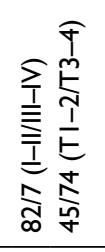 & 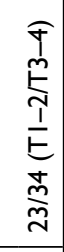 & \\
\hline है & 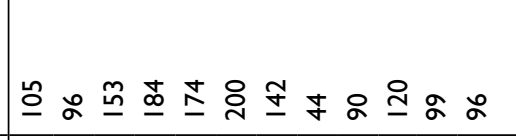 & 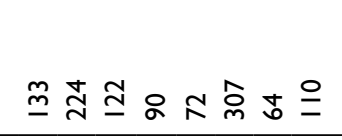 & 咅范 8 \& & $\stackrel{\infty}{m}$ & $\infty \stackrel{\circ}{=}$ & in & \\
\hline Uั & 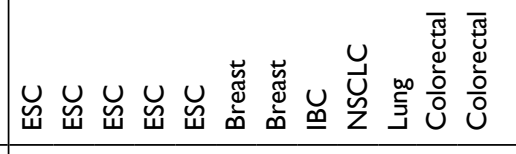 & 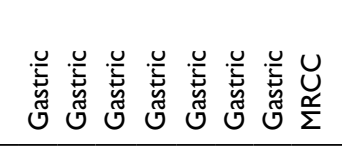 & 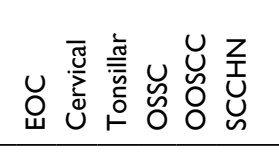 & $\sum_{\substack{0 \\
\Sigma}}$ & 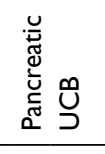 & $\stackrel{0}{\partial}$ & \\
\hline نे & 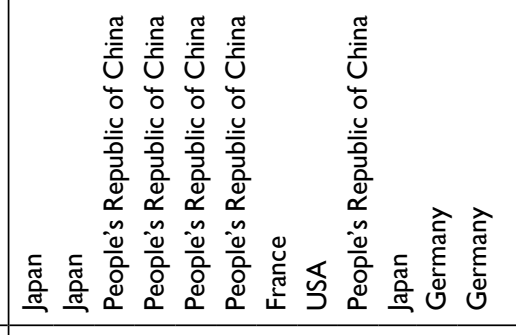 & 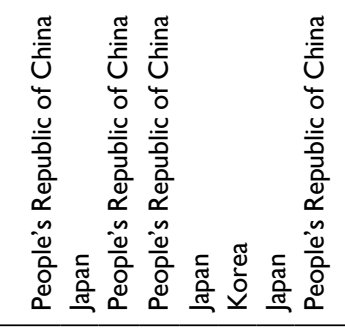 & 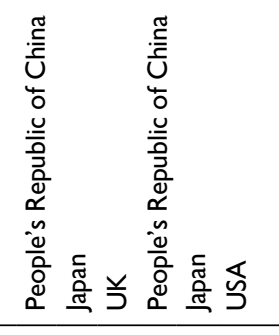 & 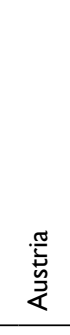 & 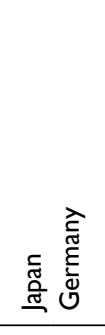 & 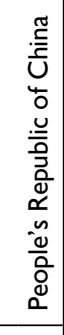 & \\
\hline ঠ & 茫 命 & 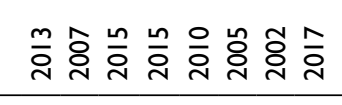 & 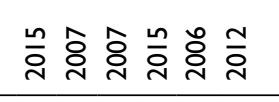 & $\frac{4}{2}$ & 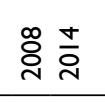 & $\frac{m}{i}$ & \\
\hline$\frac{0}{2}$ & 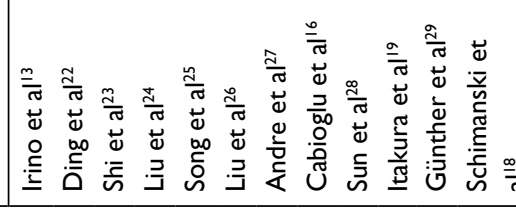 & 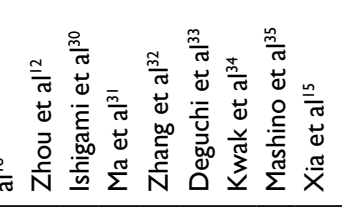 & 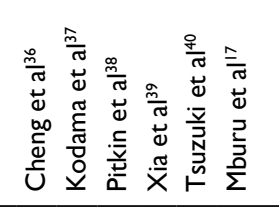 & & 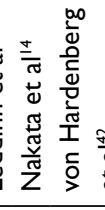 & & \\
\hline
\end{tabular}


Table 2 Pooled HRs and 95\% Cls for OS according to subgroup

\begin{tabular}{|c|c|c|c|c|c|c|}
\hline \multirow[t]{2}{*}{ Subgroup analysis } & \multirow[t]{2}{*}{ No. of patients } & \multirow[t]{2}{*}{ No. of studies } & \multicolumn{2}{|c|}{ Random-effects model } & \multicolumn{2}{|c|}{ Heterogeneity } \\
\hline & & & HR (95\% Cl) & $P$-value & $I^{2}(\%)$ & $P$-value \\
\hline OS & 3,237 & 28 & $1.79(1.49-2.16)$ & $<0.001$ & 67.8 & 0.000 \\
\hline \multicolumn{7}{|l|}{ Ethnicity } \\
\hline Asian & 2,687 & 21 & $1.89(1.56-2.29)$ & $<0.001$ & 63.0 & 0.000 \\
\hline Caucasian & 550 & 7 & $1.53(0.89-2.62)$ & 0.121 & 77.0 & 0.000 \\
\hline \multicolumn{7}{|l|}{ Tumor type } \\
\hline ESC & 712 & 5 & $2.21(1.74-2.82)$ & $<0.001$ & 0 & 0.496 \\
\hline Gastric & 1,012 & 7 & $1.99(1.58-2.51)$ & $<0.001$ & 0 & 0.954 \\
\hline Breast & 386 & 3 & $1.24(0.94-1.62)$ & 0.123 & 29.9 & 0.240 \\
\hline Lung & 210 & 2 & $0.89(0.29-2.7 I)$ & 0.841 & 92.2 & 0.000 \\
\hline Colorectal & 195 & 2 & $0.92(0.20-4.30)$ & 0.919 & 92.7 & 0.000 \\
\hline Other & 722 & 9 & $2.38(1.94-2.93)$ & $<0.001$ & 0 & 0.990 \\
\hline \multicolumn{7}{|l|}{ Tumor source } \\
\hline Digestive system & 2,008 & 15 & $1.89(1.50-2.38)$ & $<0.001$ & 55.1 & 0.005 \\
\hline Urogenital system & 314 & 3 & $2.42(1.87-3.13)$ & $<0.001$ & 0 & 0.766 \\
\hline Other & 915 & 10 & $1.50(1.09-2.06)$ & 0.012 & 68.9 & 0.001 \\
\hline \multicolumn{7}{|l|}{ Analysis type } \\
\hline Multivariate & 965 & 10 & $2.12(1.65-2.73)$ & $<0.001$ & 40 & 0.091 \\
\hline Univariate & 2,272 & 18 & $1.63(1.30-2.06)$ & $<0.001$ & 70.8 & 0.000 \\
\hline \multicolumn{7}{|l|}{ HR acquisition method } \\
\hline Reported & 1,227 & 10 & $1.64(I . \mid 2-2.4 I)$ & 0.012 & 75.0 & 0.000 \\
\hline Extrapolated & 2,010 & 18 & $1.88(1.52-2.33)$ & $<0.001$ & 64.0 & 0.000 \\
\hline
\end{tabular}

Abbreviations: ESC, esophageal squamous cell carcinoma; OS, overall survival.

\section{CCR7 and DFS}

DFS data were reported by three studies for a total of 406 patients..$^{27,37,40}$ As significant heterogeneity was found $\left(I^{2}=74.5 \% ; P=0.020\right)$ in this meta-analysis, a random-effects model was used to calculate the pooled HR and $95 \%$ CI. The pooled HR was 1.69 (95\% CI $=0.79-3.61 ; P=0.175)$, which indicated that CCR7 expression did not correlate significantly with DFS (Figure 4A).

\section{CCR7 and PFS}

Three studies comprising 192 patients provided valid data for PFS analysis. ${ }^{15,17,41}$ We used a fixed effects model to carry out the meta-analysis, as there was no obvious heterogeneity $\left(I^{2}=4.7 \% ; P=0.350\right)$. Higher expression of CCR7 was associated with significantly poorer PFS (pooled $\mathrm{HR}=2.18 ; 95 \%$ $\mathrm{CI}=1.49-3.81 ; P<0.001)$ (Figure 4B).

\section{CCR7 and RFS}

Three articles including 261 patients provided relevant data for RFS analysis. ${ }^{19,38,43}$ As significant heterogeneity was detected $\left(I^{2}=88.4 \% ; P=0.000\right)$, we used a random-effects model to calculate the pooled HR. The pooled HR was 1.29 (95\% CI $=0.48-3.44 ; P=0.618$ ), which illustrated that higher CCR7 expression tended to portend poorer RFS, but no statistical significance was found (Figure 4C).

\section{CCR7 and DSS}

For DSS, we performed a meta-analysis with two studies. ${ }^{38,42}$ A random effects model was used because heterogeneity was identified, and the pooled HR was $3.06(95 \% \mathrm{CI}=0.38-24.83$; $P=0.294)$. The detailed data are described in Figure 4D.

\section{Heterogeneity analysis}

Due to the existence of significant heterogeneity, we performed further analyses to identify potential sources of heterogeneity. First, we conducted a meta-regression analysis, and found that the tumor type ( $P=0.117)$, patient ethnicity $(P=0.349)$, analysis type ( $P=0.172)$, HR acquisition method $(P=0.573)$, sample size $(P=0.504)$, publication year ( $P=0.509)$, follow-up period $(P=0.091)$, and cutoff value $(P=0.444)$ contributed little to the heterogeneity. We also carried out subgroup analyses to explore the heterogeneity based on tumor type, tumor source, and patient ethnicity. The heterogeneity was still significant in each group (Table 2), indicating that these factors could not solely explain the heterogeneity of the OS analysis.

To evaluate the stability and credibility of the heterogeneity, we performed a sensitivity analysis by omitting individual studies from the OS analysis using a fixed effects model. No individual study obviously impacted the combined HR (Figure 5). We also conducted sensitivity analyses to determine 


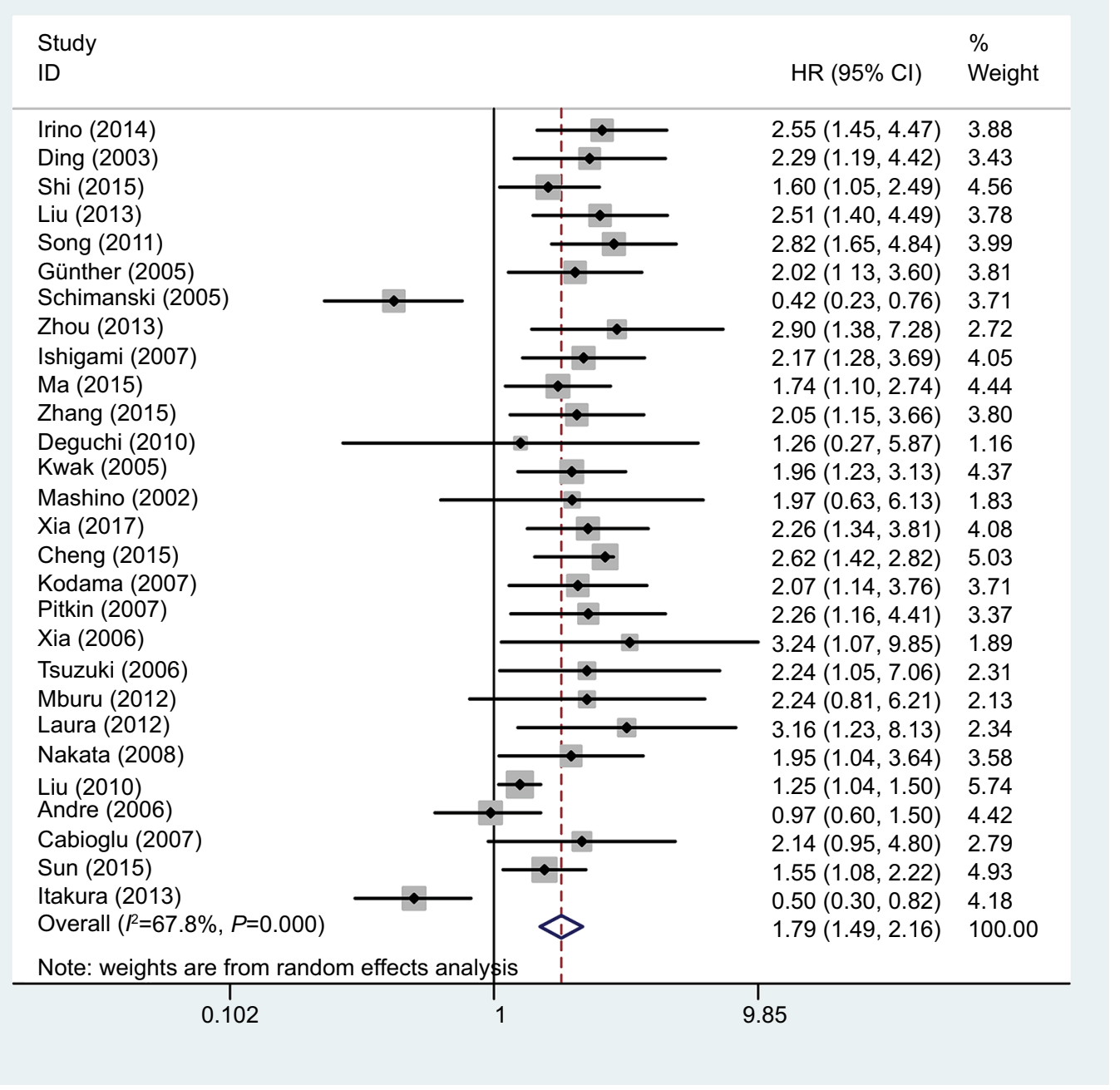

Figure 2 Forest plot of studies evaluating the effect of high CCR7 expression on the $\mathrm{HR}$ and $95 \% \mathrm{Cl}$ of OS in cancer patients. Abbreviations: CCR7, C-C chemokine receptor type 7; OS, overall survival.

possible factors contributing to the heterogeneity of the DFS and RFS analyses. We found that no single study influenced the result pattern for DFS or RFS (Figure 6A and B).

\section{Publication bias}

We evaluated the possible publication bias of the included studies by using a funnel plot and Egger's test. The funnel plot of the OS analysis is shown in Figure 7. The $P$-value of Egger's regression intercept was 0.339 , illustrating that there was no obvious publication bias in this meta-analysis. As the number of studies was not adequate to gain reliable results, we did not carry out publication bias analyses for the DFS, PFS, RFS, or DSS group.

\section{Discussion}

CCR7, one of the seven transmembrane domain G proteincoupled receptors, is the common receptor for chemokines
CCL19 and CCL21., 54 They are naturally expressed by various immune cells and secondary lymphoid tissues. The binding of CCR7 to CCL19 and CCL21 induces the lymphnode homing of naive and regulatory $\mathrm{T}$ cells and dendritic cells, and promotes the metastasis of these cells to secondary lymphoid organs. The CCR7-CCL19/CCL21 axis regulates various adaptive immune functions, including regulatory and memory T-cell function, secondary lymphoid structure formation, thymocyte genesis, lymphocyte exudation in tumor tissue, high-affinity antigen-antibody immune responses, etc. This signaling axis is also crucial for maintaining the balance between immunity and tolerance. ${ }^{45-47}$

Due to the unique biological function of CCR7 in the immune system, the influence of it on the microenvironment of tumors has attracted widespread academic attention. It is abnormally expressed in a variety of tumors, and contributes to various malignant biological behaviors of tumors, 


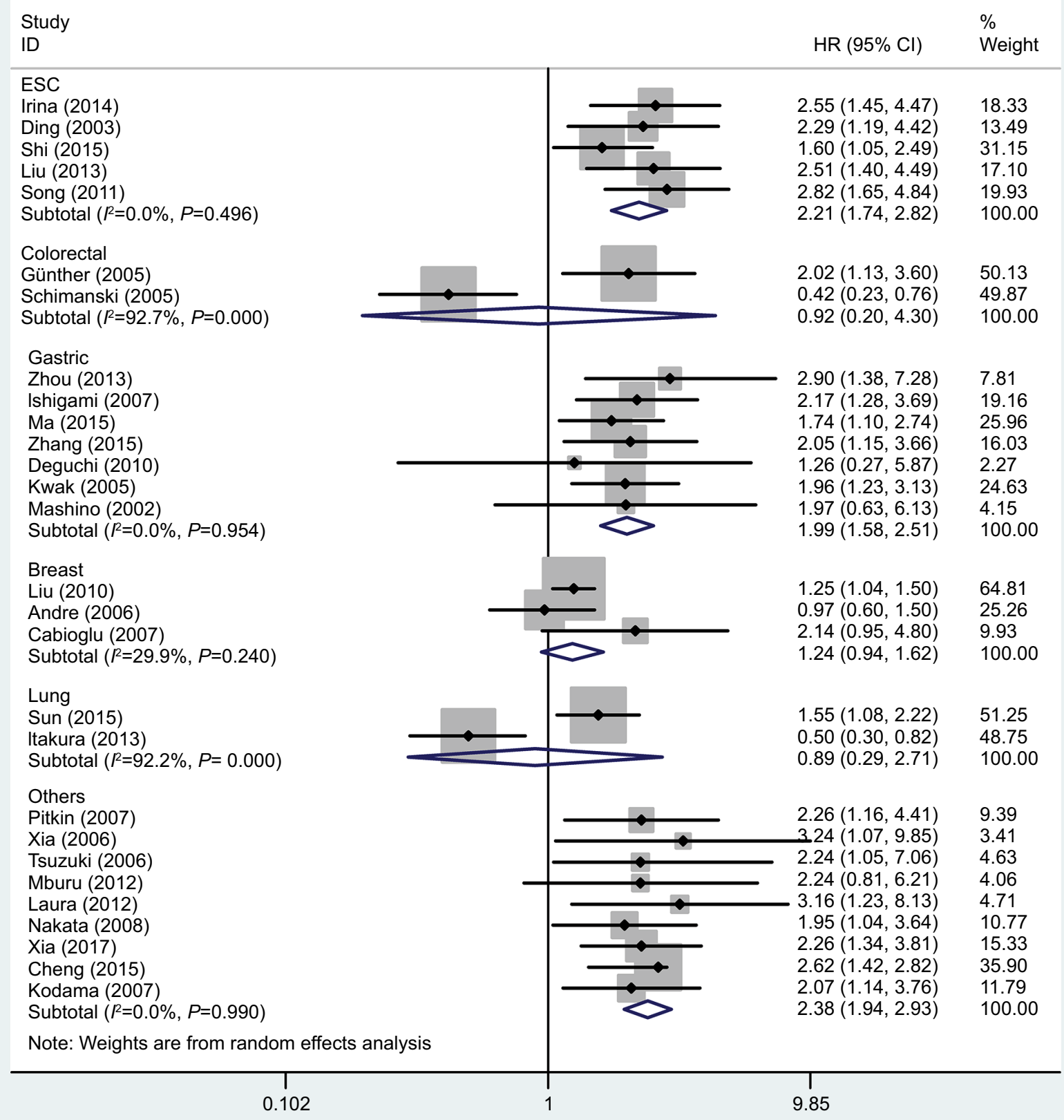

Figure 3 Forest plot of studies evaluating the relationship between high CCR7 expression and OS in patients with different cancers.

Abbreviations: CCR7, C-C chemokine receptor type 7; ESC, esophageal squamous cell carcinoma; OS, overall survival.

especially, tumor lymph node metastasis. The CCR7-CCL19/ CCL21 axis primarily promotes lymph node metastasis by recruiting tumor cells to the T-cell zone of the lymph nodes. ${ }^{47}$ The chemotactic interaction between CCR7 and CCL21 has been confirmed as the mechanism for lymph node metastasis in gastric cancer ${ }^{35}$ and esophageal cancer. ${ }^{22}$ CCR7/CCL21 is also widely recognized to induce lymph node metastasis by upregulating the expression of vascular endothelial growth factors C and D and further promoting lymphangiogenesis. ${ }^{28,48,49}$ Several recent studies have indicated that CCR7 is an important biological marker of tumor lymph node metastasis in many other cancers, including breast cancer, ${ }^{50}$ pancreatic cancer, ${ }^{51}$ bladder cancer, ${ }^{52}$ and head and neck squamous cell carcinoma. ${ }^{10}$

CCR7 is also a key regulator of tumor invasion and distant metastasis. The main regulatory mechanisms are that 


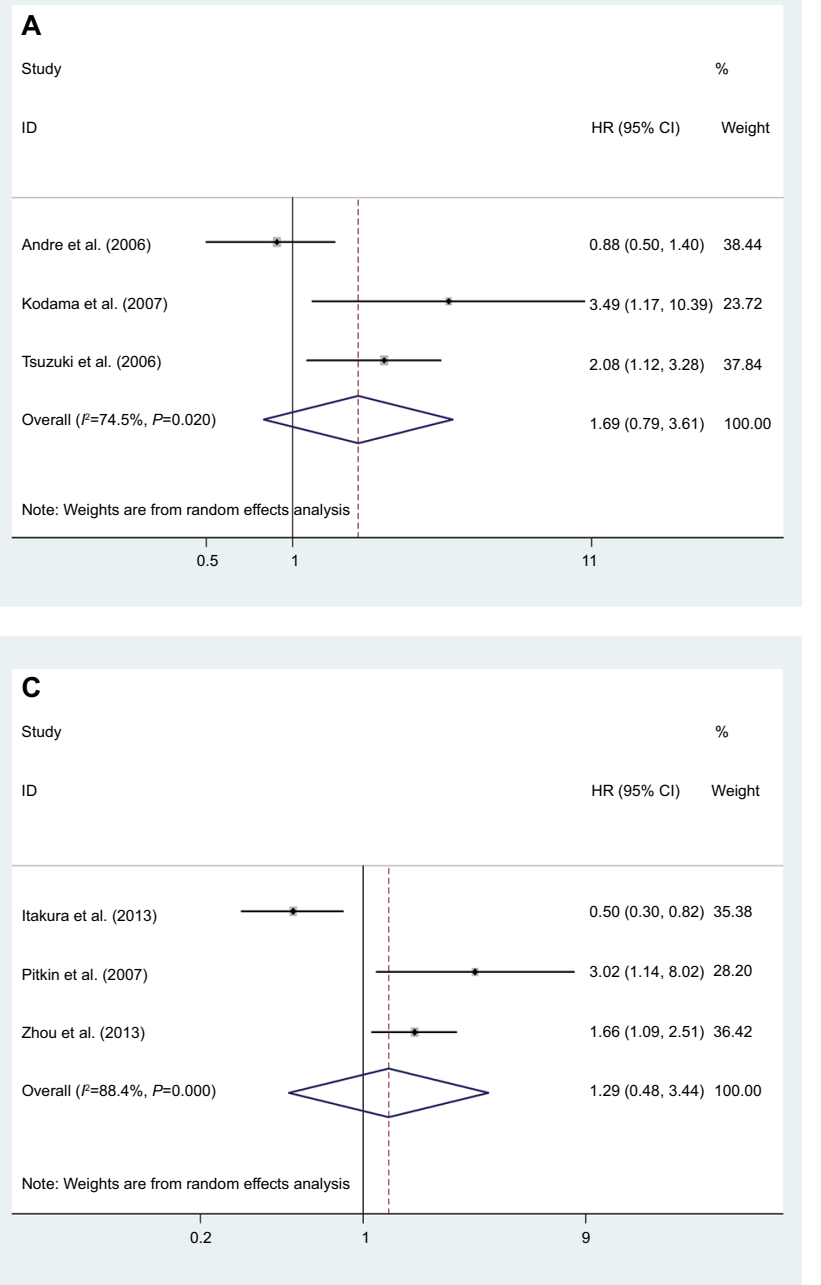

B

Study

ID

HR $(95 \% \mathrm{Cl})$ Weight

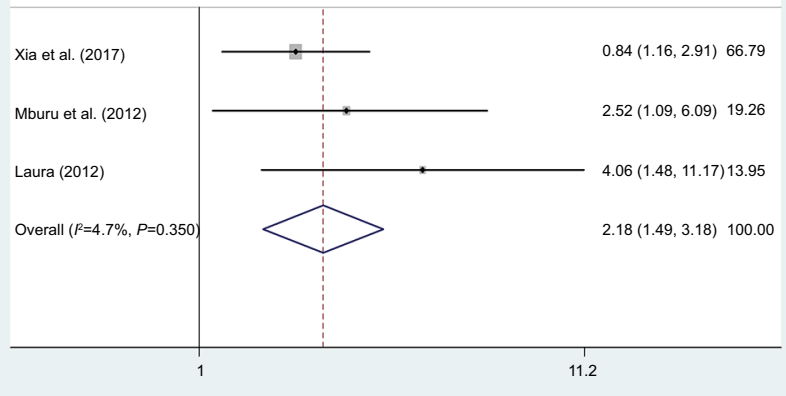

D

Study

$\%$

ID

HR $(95 \% \mathrm{Cl}) \quad$ Weight

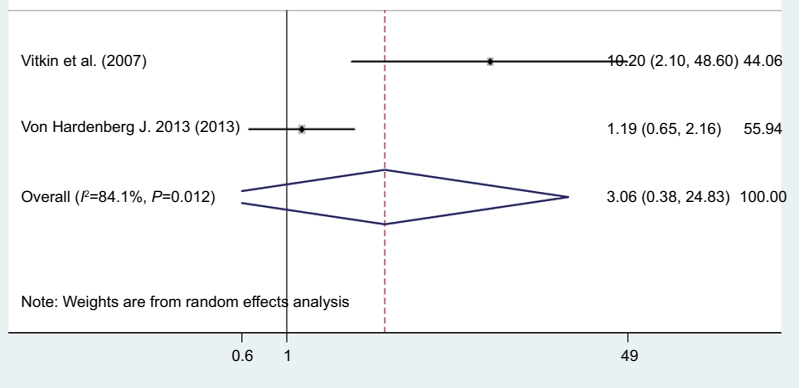

Figure 4 Forest plot of studies evaluating the effects of high CCR7 expression on the HRs and $95 \%$ Cls of different survival parameters in cancer patients. Note: Meta-analysis of (A) DFS; (B) PFS; (C) RFS; and (D) DSS.

Abbreviations: CCR7, C-C chemokine receptor type 7; DFS, disease-free survival; DSS, disease-specific survival; PFS, progress-free survival; RFS, recurrence-free survival.

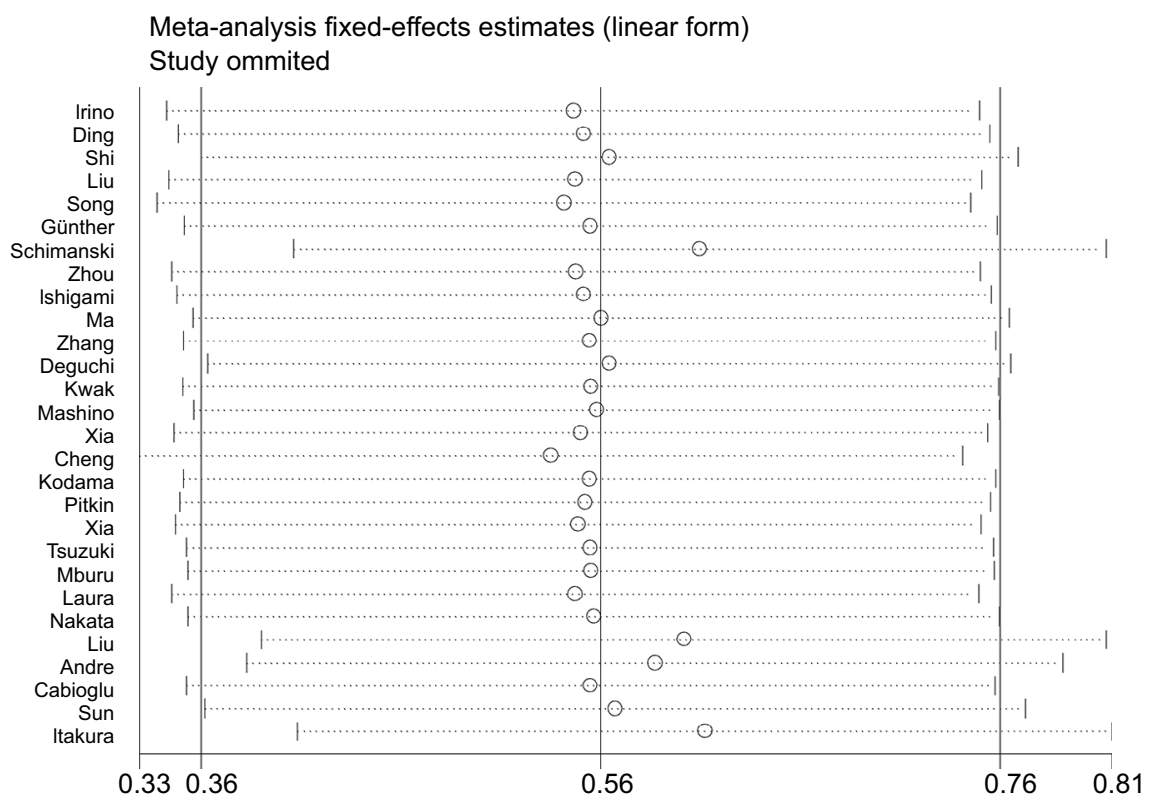

Figure 5 Forest plot of sensitivity analysis for OS.

Abbreviation: OS, overall survival. 

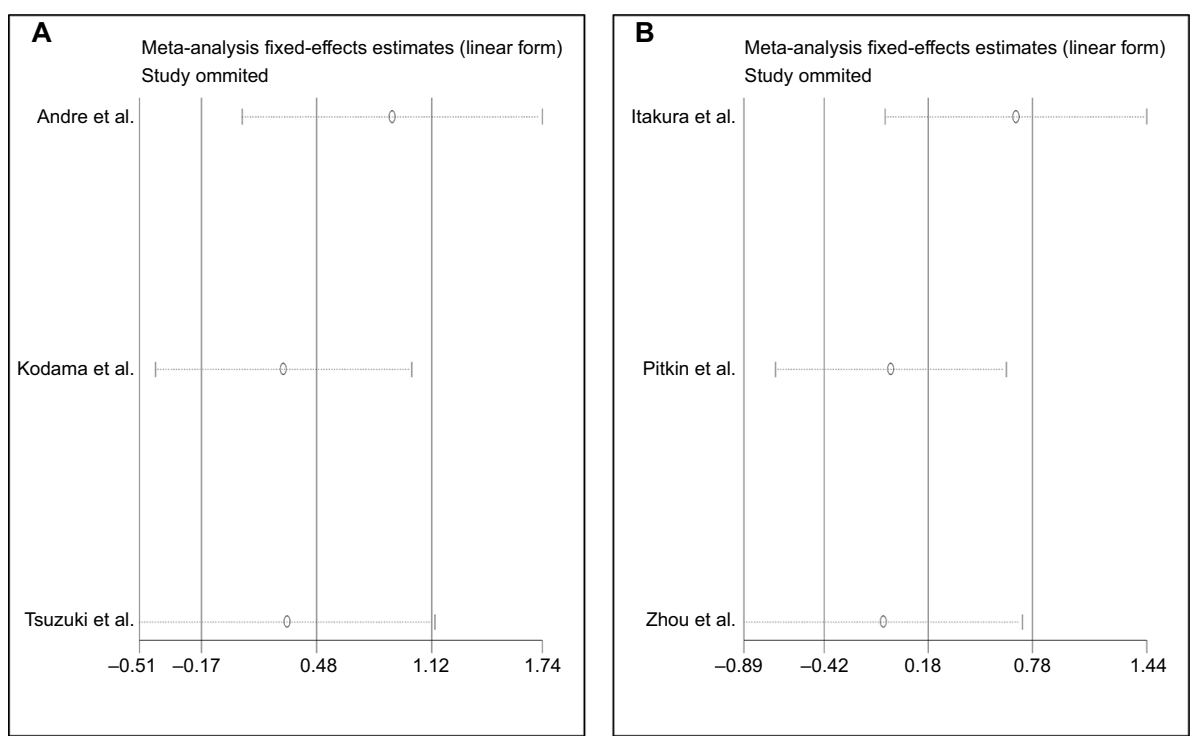

Figure 6 Forest plot of sensitivity analysis for (A) DFS and (B) RFS

Abbreviations: DFS, disease-free survival; RFS, recurrence-free survival.

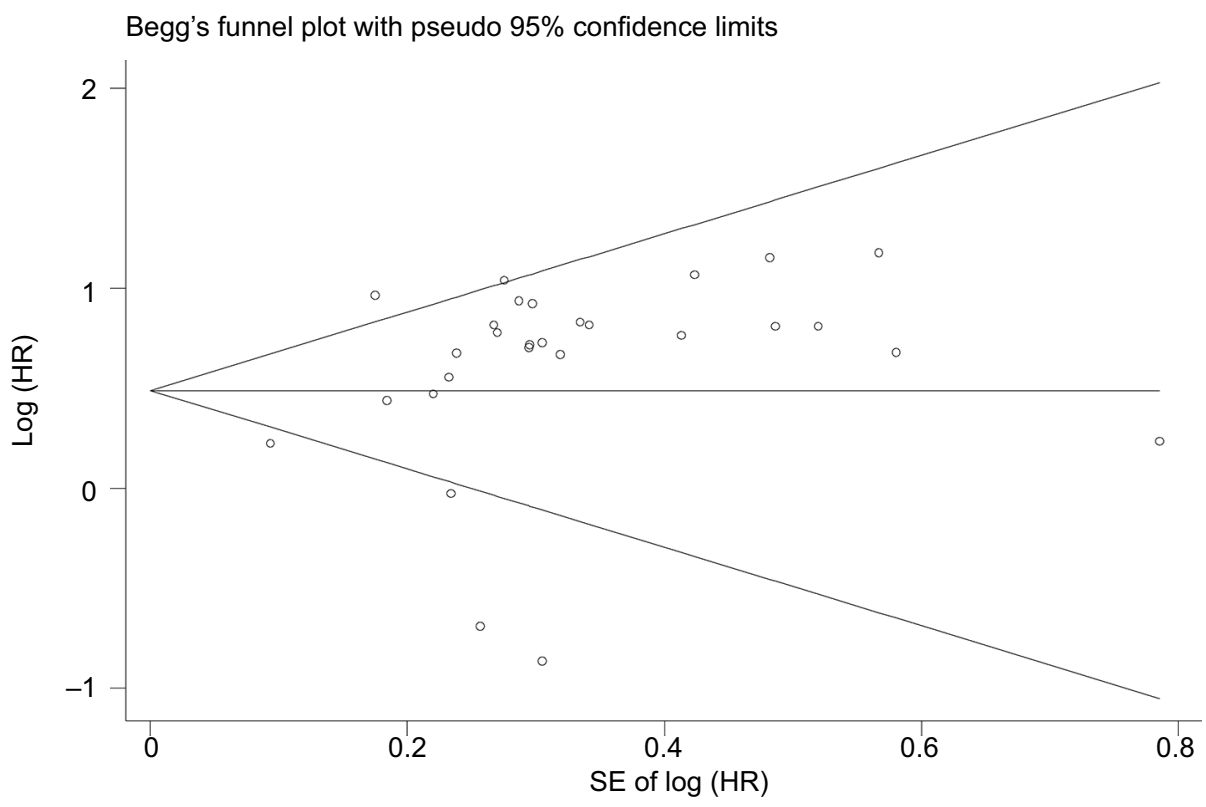

Figure 7 Funnel plot of publication bias for patients' OS.

Abbreviation: OS, overall survival.

1) the CCR7-CCL21/CCL19 axis indirectly facilitates the epithelial-mesenchymal transition by regulating upstream gene expression; ${ }^{36,53}$ 2) the CCR7-CCL21/CCL19 axis promotes the expression of matrix metalloproteinases 2 and 9, markers of malignant tumor metastasis and invasion; ${ }^{54,55}$ and 3) CCR7 induces phosphatidylinositol-3 kinase/protein kinase B signaling, ${ }^{56}$ mitogen-activated protein kinase/ extracellular signal-regulated kinase $1 / 2$ signaling, ${ }^{57}$ and other related signaling pathways that promote tumor invasion and metastasis. An adequate blood supply is essential for tumor progression, and CCR7 also greatly stimulates tumor angiogenesis, mainly through the following mechanisms: 1) increasing the microvessel density, as part of the CCL21/ CCR7 axis in tumor tissues; ${ }^{11}$ 2) enhancing the angiogenic capacity and inducing the proliferation and migration of human umbilical vein endothelial cells by activating the nuclear factor $\mathrm{\kappa B}$ pathway ${ }^{58}$ and 3 ) promoting the expression of vascular endothelial growth factor $\mathrm{C}$ in tumor tissues. ${ }^{59}$ In 
addition, CCR7 has been reported to prevent apoptosis and induce proliferation by promoting G2/M phase progression via the extracellular signal-regulated kinase pathway in human NSCLC. ${ }^{9,60}$ Moreover, CCR7 can downregulate interferon $\gamma$-induced inflammatory gene expression to weaken the host's antitumor immunity, ${ }^{61}$ thus facilitating cancer cell dissemination. ${ }^{44}$ Therefore, CCR7 overexpression in the tumor microenvironment is important for cancer progression and is associated with poor prognoses in cancer patients.

Although several studies have shown that CCR7 is a reliable indicator for the prognosis of tumor patients, the effect of CCR7 on the OS of tumor patients remains controversial. Different studies have had the opposite results, even regarding the OS of patients with the same tumor type, such as colorectal cancer ${ }^{23,24}$ or lung cancer. ${ }^{19,28}$ In addition, CCR7 has been reported to have no significant effect on OS in some tumor types, such as gastric cancer, ${ }^{33,35}$ breast cancer, ${ }^{16,27}$ and SCCHN. ${ }^{17}$ We found that the relationship between CCR7 and the prognosis of various tumors had not been systematically reviewed and further evaluated. Thus, we conducted this meta-analysis to provide a reliable evidence-based medical resource on the prognostic value of CCR7. In total, 30 studies encompassing 3,413 patients with 15 different types of tumors were enrolled in our meta-analysis, and five survival parameters (OS, PFS, RFS, DFS, and DSS) were analyzed. Through this meta-analysis, we provided strong evidence that higher expression of CCR7 is an independent prognostic indicator of poorer OS in patients with solid tumors. Elevated CCR7 levels were also associated with significantly worse PFS; however, there were not sufficient data to confirm that CCR7 was associated with DFS, RFS, or DSS.

To further evaluate the prognostic value of CCR7 in different tumors, we conducted subgroup analyses for OS. Due to the limited number of available articles, we did not carry out subgroup analyses for DFS, RFS, PFS, or DSS. We found that the upregulation of CCR7 significantly shortened the OS of patients with esophageal cancer and gastric cancer. In addition, overexpression of CCR7 tended to predict worse OS in breast cancer patients, but this impact was not notable. Remarkably, CCR7 had the opposite effect on patients with lung cancer and colorectal cancer, as higher CCR7 expression tended to be associated with a better prognosis; however, the results were not statistically significant. For these results, we cannot exclude the impact of insufficient sample sizes and limitations of the studies. We also found that CCR7 was a negative prognostic factor for OS in patients with digestive system and urogenital system tumors. In other systems, however, the relationship between CCR7 expression and tumor prognosis was not clear, suggesting that larger sample sizes and prospective studies are needed to further investigate the clinical prognostic value of CCR7 according to the tumor source.

We also explored the influence of CCR7 on the OS of patients from different ethnic backgrounds. Patients were categorized as Asian or Caucasian according to the country in which the study was conducted. Different combined HRs and $P$-values for OS were found in Asian and Caucasian patients: the association of high CCR7 expression with poor OS was more significant for Asian patients than for Caucasians. This suggests that racial differences may be an objective factor influencing the survival rate of tumor patients.

Although this meta-analysis confirmed the tumor prognostic value of CCR7, the limitations of this study should still be acknowledged. First, only 30 studies were enrolled in this meta-analysis, so there was a lack of relevant data for correlative and subgroup analyses. Second, the cutoff value for CCR7 expression was not uniform across the studies, which may reduce the effectiveness of CCR7 as a tumor prognostic marker. Third, some of the HR values were extracted from survival curves, which inevitably produced small statistical errors. Finally, this meta-analysis exhibited significant heterogeneity, which could be attributed to several factors, such as the tumor type, analysis method, sample source, cutoff value, follow-up period, and publication year.

In summary, our meta-analysis has suggested that in most solid malignancies, higher expression of CCR7 is associated with poorer prognoses of patients. Although elevated CCR7 expression tended to predict better prognoses in patients with lung cancer and colorectal cancer, these results need to be further confirmed due to their lack of statistical significance. Thus, we can conclude that CCR7 is a reliable, valuable prognostic marker in cancers. In view of the limitations of the current research, this conclusion should be interpreted cautiously. It will be necessary to design large, high-quality, multicenter, prospective clinical trials to confirm the potential value of CCR7 for the prognostic determination, clinical diagnosis, and treatment of tumor patients.

\section{Author contributions}

GZ and BL contributed equally to this work. YY and YT designed the research. GZ and BL wrote the manuscript. GZ, BL, YY, and YT collected the relevant papers and data. GZ, $\mathrm{BL}, \mathrm{YY}$, and TT analyzed and processed the data. All authors contributed to data analysis, drafting or revising the article, gave final approval of the version to be published, and agree to be accountable for all aspects of the work.

\section{Disclosure}

The authors report no conflicts of interest in this work. 


\section{References}

1. Siegel RL, Miller KD, Jemal A. Cancer statistics, 2016. CA Cancer J Clin. 2016;66(1):7-30.

2. Miller KD, Siegel RL, Lin CC, et al. Cancer treatment and survivorship statistics, 2016. CA Cancer J Clin. 2016;66(4):271-289.

3. Balkwill FR. The chemokine system and cancer. J Pathol. 2012;226(2):148-157.

4. Müller A, Homey B, Soto $\mathrm{H}$, et al. Involvement of chemokine receptors in breast cancer metastasis. Nature. 2001;410(6824):50-56.

5. Ott TR, Pahuja A, Nickolls SA, Alleva DG, Struthers RS. Identification of CC chemokine receptor 7 residues important for receptor activation. J Biol Chem. 2004;279(41):42383-42392.

6. Willimann K, Legler DF, Loetscher M, et al. The chemokine SLC is expressed in $\mathrm{T}$ cell areas of lymph nodes and mucosal lymphoid tissues and attracts activated T cells via CCR7. Eur J Immunol. 1998;28(6):2025-2034.

7. Hirao M, Onai N, Hiroishi K, et al. CC chemokine receptor-7 on dendritic cells is induced after interaction with apoptotic tumor cells: critical role in migration from the tumor site to draining lymph nodes. Cancer Res. 2000;60(8):2209-2217.

8. Dieu MC, Vanbervliet B, Vicari A, et al. Selective recruitment of immature and mature dendritic cells by distinct chemokines expressed in different anatomic sites. J Exp Med. 1998;188(2):373-386.

9. Xu Y, Liu L, Qiu X, et al. CCL21/CCR7 promotes G2/M phase progression via the ERK pathway in human non-small cell lung cancer cells. PLoS One. 2011;6(6):e21119.

10. Liu FY, Safdar J, Li ZN, et al. CCR7 regulates cell migration and invasion through JAK2/STAT3 in metastatic squamous cell carcinoma of the head and neck. Biomed Res Int. 2014;2014(4):1-11.

11. Zhao B, Cui K, Wang CL, et al. The chemotactic interaction between CCL21 and its receptor, CCR7, facilitates the progression of pancreatic cancer via induction of angiogenesis and lymphangiogenesis. J Hepatobiliary Pancreat Sci. 2011;18(6):821-828.

12. Zhou S, Shen Z, Wang Y, et al. CCR7 expression and intratumoral Foxp3+ regulatory $T$ cells are correlated with overall survival and lymph node metastasis in gastric cancer. PLoS One. 2013;8(9):e74430.

13. Irino $\mathrm{T}$, Takeuchi $\mathrm{H}$, Matsuda $\mathrm{S}$, et al. CC-chemokine receptor CCR7: a key molecule for lymph node metastasis in esophageal squamous cell carcinoma. BMC Cancer. 2014;14:291.

14. Nakata B, Fukunaga S, Noda E, Amano R, Yamada N, Hirakawa K. Chemokine receptor CCR7 expression correlates with lymph node metastasis in pancreatic cancer. Oncology. 2008;74(1-2):69-75.

15. Xia Y, Liu L, Xiong Y, et al. Prognostic value of CC-chemokine receptor seven expression in patients with metastatic renal cell carcinoma treated with tyrosine kinase inhibitor. BMC Cancer. 2017;17(1):70.

16. Cabioglu N, Gong Y, Islam R, et al. Expression of growth factor and chemokine receptors: new insights in the biology of inflammatory breast cancer. Ann Oncol. 2007;18(6):1021-1029.

17. Mburu YK, Egloff AM, Walker WH, et al. Chemokine receptor 7 (CCR7) gene expression is regulated by NF- $\mathrm{KB}$ and activator protein 1 (AP1) in metastatic squamous cell carcinoma of head and neck (SCCHN). J Biol Chem. 2012;287(5):3581-3590.

18. Schimanski CC, Schwald S, Simiantonaki N, et al. Effect of chemokine receptors CXCR4 and CCR7 on the metastatic behavior of human colorectal cancer. Clin Cancer Res. 2005;11(5):1743-1750.

19. Itakura M, Terashima Y, Shingyoji M, et al. High CC chemokine receptor 7 expression improves postoperative prognosis of lung adenocarcinoma patients. Br J Cancer. 2013;109(5):1100-1108.

20. Moher D, Liberati A, Tetzlaff J, Altman DG; PRISMA Group. Preferred reporting items for systematic reviews and meta-analyses: the PRISMA statement. Int J Surg. 2010;8(5):336-341.

21. Stroup DF, Berlin JA, Morton SC, et al. Meta-analysis of observational studies in epidemiology: a proposal for reporting. Meta-analysis of observational studies in epidemiology (moose) group. JAMA. 2000;283(15):2008-2012.

22. Ding Y, Shimada Y, Maeda M, et al. Association of CC chemokine receptor 7 with lymph node metastasis of esophageal squamous cell carcinoma. Clin Cancer Res. 2003;9(9):3406-3412.
23. Shi M, Chen D, Yang D, Liu XY. CCL21-CCR7 promotes the lymph node metastasis of esophageal squamous cell carcinoma by up-regulating MUC1. J Exp Clin Cancer Res. 2015;34(1):149.

24. Liu XY, Song L, Wang Z. CCR7: a metastasis and prognosis indicator of postoperative patients with esophageal carcinoma. Hepatogastroenterology. 2013;60(124):747-750.

25. Song L, Wang Z, Liu X, Chen G, Liu F. Expression of CCR7 in squamous cell carcinoma of the esophagus after esophagectomy: correlation with lymph node metastasis and prognosis. Chin J Thorac Cardiovasc Surg. 2011;27:361-364.

26. Liu Y, Ji R, Li J, et al. Correlation effect of EGFR and CXCR4 and CCR7 chemokine receptors in predicting breast cancer metastasis and prognosis. J Exp Clin Cancer Res. 2010;29(1):16.

27. Andre F, Cabioglu N, Assi H, et al. Expression of chemokine receptors predicts the site of metastatic relapse in patients with axillary node positive primary breast cancer. Ann Oncol. 2006;17(6):945-951.

28. Sun L, Zhang Q, Li Y, Tang N, Qiu X. CCL21/CCR7 up-regulate vascular endothelial growth factor-D expression via ERK pathway in human non-small cell lung cancer cells. Int J Clin Exp Pathol. 2015;8(12):15729-15738.

29. Günther K, Leier J, Henning G, et al. Prediction of lymph node metastasis in colorectal carcinoma by expressionof chemokine receptor CCR7. Int J Cancer. 2005;116(5):726-733.

30. Ishigami S, Natsugoe S, Nakajo A, et al. Prognostic value of CCR7 expression in gastric cancer. Hepatogastroenterology. 2007;54(76):1025-1028.

31. Ma H, Gao L, Li S, et al. CCR7 enhances TGF- $\beta 1$-induced epithelial-mesenchymal transition and is associated with lymph node metastasis and poor overall survival in gastric cancer. Oncotarget. 2015;6(27):24348-24360.

32. Zhang J, Zhou Y, Yang Y. CCR7 pathway induces epithelial-mesenchymal transition through up-regulation of snail signaling in gastric cancer. Med Oncol. 2015;32(2):467.

33. Deguchi K, Ichikawa D, Soga K, et al. Clinical significance of vascular endothelial growth factors $\mathrm{C}$ and D and chemokine receptor CCR7 in gastric cancer. Anticancer Res. 2010;30(6):2361-2366.

34. Kwak MK, Hur K, Park DJ, et al. Expression of chemokine receptors in human gastric cancer. Tumor Biol. 2005;26(2):65-70.

35. Mashino K, Sadanaga N, Yamaguchi H, et al. Expression of chemokine receptor CCR7 is associated with lymph node metastasis of gastric carcinoma. Canc Res. 2002;62(10):2937-2941.

36. Cheng S, Guo J, Yang Q, Yang X. Crk-like adapter protein regulates CCL19/ CCR7-mediated epithelial-to-mesenchymal transition via ERK signaling pathway in epithelial ovarian carcinomas. Med Oncol. 2015;32(3):47.

37. Kodama J, Hasengaowa, Seki N, Kusumoto T, Hiramatsu Y. Expression of the CXCR4 and CCR7 chemokine receptors in human endometrial cancer. Eur J Gynaecol Oncol. 2007;28(5):370-375.

38. Pitkin L, Luangdilok S, Corbishley C, et al. Expression of CC chemokine receptor 7 in tonsillar cancer predicts cervical nodal metastasis, systemic relapse and survival. Br J Cancer. 2007;97(5):670-677.

39. Xia X, Liu K, Zhang H, Shang Z. Correlation between CCR7 expression and lymph node metastatic potential of human tongue carcinoma. Oral Dis. 2015;21(1):123-131.

40. Tsuzuki H, Takahashi N, Kojima A, et al. Oral and oropharyngeal squamous cell carcinomas expressing CCR7 have poor prognoses. Auris Nasus Larynx. 2006;33(1):37-42.

41. Kühnelt-Leddihn L, Müller H, Eisendle K, Zelger B, Weinlich G. Overexpression of the chemokine receptors CXCR4, CCR7, CCR9, and CCR10 in human primary cutaneous melanoma: a potential prognostic value for CCR7 and CCR10? Arch Dermatol Res. 2012;304(3):185-193.

42. von Hardenberg J, Martini T, Knauer A, et al. Expression and predictive value of lymph-specific markers in urothelial carcinoma of the bladder. Urol Oncol. 2014;32(1):54.e9-17.

43. Zhou M, Zhang D, Xiaolong Q, Liu F, Lin Q, Xiongbing Z. Significance of CCR7 expression in bladder urothelial carcinoma. Chin J Urol. 2013;34(5):352-356.

44. Legler DF, Uetz-von Allmen E, Hauser MA. CCR7: roles in cancer cell dissemination, migration and metastasis formation. Int J Biochem Cell Biol. 2014;54:78-82. 
45. Förster R, Davalos-Misslitz AC, Rot A. CCR7 and its ligands: balancing immunity and tolerance. Nat Rev Immunol. 2008;8(5):362-371.

46. Comerford I, Harata-Lee Y, Bunting MD, Gregor C, Kara EE, McColl SR. A myriad of functions and complex regulation of the CCR7/CCL19/ CCL21 chemokine axis in the adaptive immune system. Cytokine Growth Factor Rev. 2013;24(3):269-283.

47. Zlotnik A, Burkhardt AM, Homey B. Homeostatic chemokine receptors and organ-specific metastasis. Nat Rev Immunol. 2011;11(9):597-606.

48. Tutunea-Fatan E, Majumder M, Xin X, Lala PK. The role of CCL21/ CCR7 chemokine axis in breast cancer-induced lymphangiogenesis. Mol Canc. 2015;14(1):35.

49. Song Y, Wang Z, Liu X, Jiang W, Shi M. CCR7 and VEGF-C: molecular indicator of lymphatic metastatic recurrence in pN0 esophageal squamous cell carcinoma after Ivor-Lewis esophagectomy? Ann Surg Oncol. 2012;19(11):3606-3612.

50. El-Ghonaimy EA, El-Shinawi M, Ibrahim SA, et al. Positive lymphnode breast cancer patients - activation of NF- $\mathrm{\kappa B}$ in tumor-associated leukocytes stimulates cytokine secretion that promotes metastasis via C-C chemokine receptor CCR7. FEBS J. 2015;282(2):271-282.

51. Guo J, Lou W, Ji Y, Zhang S. Effect of CCR7, CXCR4 and VEGF-C on the lymph node metastasis of human pancreatic ductal adenocarcinoma. Oncol Lett. 2013;5(5):1572-1578.

52. Chen J, Cui YU, Liu L, et al. CCR7 as a predictive biomarker associated with computed tomography for the diagnosis of lymph node metastasis in bladder carcinoma. Oncol Lett. 2016;11(1):735-740.

53. Zhong G, Chen L, Yin R, et al. Chemokine (C-C motif) ligand 21/C-C chemokine receptor type 7 triggers migration and invasion of human lung cancer cells by epithelialmesenchymal transition via the extracellular signalregulated kinase signaling pathway. Mol Med Rep. 2017;15(6):4100-4108.
54. Guo N, Liu F, Yang L, Huang J, Ding X, Sun C. Chemokine receptor 7 enhances cell chemotaxis and migration of metastatic squamous cell carcinoma of head and neck through activation of matrix metalloproteinase-9. Oncol Rep. 2014;32(2):794-800.

55. Mo M, Zhou M, Wang L, et al. CCL21/CCR7 enhances the proliferation, migration, and invasion of human bladder cancer T24 cells. PLoS One. 2015;10(3): $\mathrm{e} 0119506$.

56. Li K, Xu B, Xu G, Liu R. CCR7 regulates twist to induce the epithelialmesenchymal transition in pancreatic ductal adenocarcinoma. Tumor Biol. 2016;37(1):419-424.

57. Liu FY, Safdar J, Li ZN, et al. CCR7 regulates cell migration and invasion through MAPKs in metastatic squamous cell carcinoma of head and neck. Int J Oncol. 2014;45(6):2502-2510.

58. Cai QY, Liang GY, Zheng YF, Tan QY, Wang RW, Li K. CCR7 enhances the angiogenic capacity of esophageal squamous carcinoma cells in vitro via activation of the NF- $\mathrm{\kappa B} / \mathrm{VEGF}$ signaling pathway. Am J Transl Res. 2017;9(7):3282-3292.

59. Chi BJ, du CL, Fu YF, Zhang YN, Wang RW. Silencing of CCR7 inhibits the growth, invasion and migration of prostate cancer cells induced by VEGFC. Int J Clin Exp Pathol. 2015;8(10):12533-12540.

60. Xu Y, Liu L, Qiu X, et al. CCL21/CCR7 prevents apoptosis via the ERK pathway in human non-small cell lung cancer cells. PLoS One. 2012;7(3):e33262.

61. Takekoshi T, Fang L, Paragh G, Hwang ST. CCR7-expressing B16 melanoma cells downregulate interferon- $\gamma$-mediated inflammation and increase lymphangiogenesis in the tumor microenvironment. Oncogenesis. 2012;1(5):e9.

\section{Cancer Management and Research}

\section{Publish your work in this journal}

Cancer Management and Research is an international, peer-reviewed open access journal focusing on cancer research and the optimal use of preventative and integrated treatment interventions to achieve improved outcomes, enhanced survival and quality of life for the cancer patient. The manuscript management system is completely online and includes

\section{Dovepress}

a very quick and fair peer-review system, which is all easy to use. Visit http://www.dovepress.com/testimonials.php to read real quotes from published authors. 